\title{
Schlußwort
}

\section{G. Penkert}

Neurochirurgische Abteilung (Leiter: Prof. Dr. H. Finkemeyer) des Allgemeinen Krankenhauses Altona, Paul-Ehrlich-Straße 1, D-2000 Hamburg 50

Die von Prof. Sandmann angeführten Einwände haben sicher ihre Berechtigung. Allerdings zeigt sich auch eine deutlich unterschiedliche Haltung bei gefäßchirurgischem gegenüber mikrochirurgischem Vorgehen. Die. „technisierte Gesichtsfeldeinschränkung" führt zu ciner Verkleinerung von Zugangsweg und Narbe Das mikrochirurgische Vorgehen am peripheren Nerven stellt keine Operationserschwernis dar.
Hervorzuheben ist, daß die Fallbeispiele über eine ausgeprägte neurologische Symptomatik mit chronischem Verlauf und nicht über vaskuläre Symptome verfügten. Die Angiographie der A subclavia diente lediglich der Lokalisation des Engpasses, nicht dem Nachweis der vaskulären Enge. Demnach hatte sich bei der Therapie das Gewicht auf die mikrochirurgische Neurolyse am fibrotischen peripheren Nervengewebe konzentriert. Auf Literatur, die diese Überlegung stützt, wurde verwiesen.
23. Tagung der Österreichischen Gesellschaft für Chirurgie vam 10. bis 12. Juni 1982 in Wrien, Kongreßzentrum Hofburg

Themen: 1. Die operative Behandlung gutartiger anorektaler Erkrankungen.

2. Die operative Behandlung der Verletzungen der Wirbelsäule und ihrer Folgen.

Europäisches Thema: Die chirurgische Behandlung der Lebermetastasen.

Auskunft: Sekretariat der I. Chirurg. Univ.-Klinik, A-1090 Wien, Alser Straße 4.

Fachausstellung: Med. Ausstellungs- u. Werbegesellschaft, M. Rodler \& $\mathrm{Co}, \mathrm{A}-1010 \mathrm{Wien}$, Freyung 6.

Deutscbe Gesellschaft fuir Chirurgie vom 14. bis 17. April 1982 in München

Auskunft: Prof. Dr. med. S. W'eller, BG-Unfallklinik, Rosenauer Weg 95, D-7400 Tübingen.

5. Kongreß der Europäiscben Vereinigung für Urologie vom 12. bis 15. Mai 1982 in Wien

Auskunft: Sekretariat - 5. Kongreß der Europäischen Vereinigung für Urologie, c'o Wiener Medizinische Akademie, Alser Straße 4, A-1090 Wien.
23. Tagung der Südwestdeutschen Gesellschaft für Urologie vom 20. bis 22. Mai 1982 in Pforaheim

Auskunft: Prof. Dr. med D. Völter, Lirolog. Abreilung, Krankenhaus St. Trudpert, Wolfsbergallee 50, D-7530 Pforzheim.

9. Symposium der Cbirurgiscben Arbeitsgemeinschaft Endoskopie (CAE) der Deutschen Gesellscbaft für Cbirurgie, 21. und 22. Mai 1982 in Solzburg

Thema: Diagnostik und Therapie von Erkrankungen des Pankreas (Schwerpunkt Endoskopie).

Auskünfte und Anfragen: Sekretariat der L chirurgischen Abteilung der Landeskrankenanstalten Salzburg (llll 62 22) 315 81, Div. 2352.

129. Tagung der Vereinigung Nordwestdeutscher Chirurgen unm 3. bis 5. Juni 1982 in Hildesbeim

Auskunft: Prof. Dr. med D. Borm, Chirurg. Klinik St. Bernward-Krankenhaus, Treibestraße 9, D-3200 Hildesheim.

IXth Biennial Congress of the International Society of University Colon and Rectal Surgeons vom 6. bis 10. Juni 1982 in Müncben

Auskunft: Prof. Dr. F. P. Gall, Chirurg. Univ.-Klinik, Maximiliansplatz, D-8520 Erlangen. 